\title{
ON THE NUMBER OF MINIMUM CUTS IN A GRAPH*
}

\author{
L. SUNIL CHANDRAN ${ }^{\dagger}$ AND L. SHANKAR RAM ${ }^{\ddagger}$
}

\begin{abstract}
We relate the number of minimum cuts in a weighted undirected graph with various structural parameters of the graph. In particular, we provide upper bounds for the number of minimum cuts in terms of the radius, diameter, minimum degree, maximum degree, chordality, girth, and some other parameters of the graph.
\end{abstract}

Key words. minimum cuts, circular partition

AMS subject classifications. 05C35, 05C99

DOI. $10.1137 / \mathrm{S} 0895480103427138$

1. Introduction. Let $G=(V, E)$ be a graph or a multigraph with positive weights on its edges. In this paper, we will use $n$ to denote $|V|$. By an unweighted graph, we mean that all the edges have unit weight. Let $(A, \bar{A})$ denote a cut of $G$, defined by the subsets $A \subset V$ and $\bar{A}=V-A$. We denote by $E(A, \bar{A})$ the set of edges in the cut, i.e., $E(A, \bar{A})=\{(u, v) \in E: u \in A$ and $v \in \bar{A}\}$. The weight of the cut $(A, \bar{A})$ is defined as the sum of weights on all the edges in $E(A, \bar{A})$, and will be denoted by $w(A, \bar{A})$. A minimum cut $(S, \bar{S})$ is one with the minimum weight over all cuts in $G$. (Some authors use the words global minimum cuts or connectivity cuts instead of minimum cuts). We will denote the weight of the minimum cut in $G$ by $\lambda(G)$. Note that if $G$ is unweighted, $\lambda(G)$ is the same as the edge connectivity of the graph, i.e., the minimum number of edges whose removal disconnects the graph.

Note that the minimum cut in a graph may not be unique. We use $\Lambda(G)$ to denote the number of minimum cuts in $G$. The problem of counting the number of minimum cuts in a weighted undirected graph arises in various aspects of network reliability, like testing the super- $\lambda$-ness of a graph [8], estimating the probabilistic connectedness of a stochastic graph in which edges are subject to failure with probability $p[4,5$, $6,30]$, and other areas [29]. For example, for a sufficiently small $p$, the probabilistic connectedness of $G$ can be approximated as $P(G, p) \approx 1-\Lambda(G) p^{\lambda(G)}(1-p)^{|E|-\lambda(G)}$, suggesting the importance of counting and bounding $\Lambda(G)$.

It is well known that for any weighted (positive weights) graph $G, \Lambda(G) \leq\left(\begin{array}{l}n \\ 2\end{array}\right)$, and this upper bound is achieved if $G$ is a cycle $C_{n}$ of $n$ nodes with each edge having weight $\frac{\lambda(G)}{2}[12,7,22]$. It is interesting to explore whether there exist tighter bounds for $\Lambda(G)$ when the graph satisfies various properties. For example, Bixby [7] studies $\Lambda(G)$ in terms of the weight of the minimum cuts $\lambda(G)$ in the special case where all the edge weights are positive integers and $\lambda(G)$ is an odd integer. For this case, Bixby [7] shows that $\Lambda(G) \leq\left\lfloor\frac{3 n}{2}\right\rfloor-2$. In the case of unweighted simple graphs it is shown by Lehel, Maffray, and Preissmann [23] that if $\lambda(G)=k$, where $k \geq 4$ is an even positive

*Received by the editors May 1, 2003; accepted for publication (in revised form) December 17, 2003; published electronically August 19, 2004. A preliminary version of this paper appeared in Proceedings of the 8th International Computing and Combinatorics Conference, Lecture Notes in Comput. Sci. 2387, Springer-Verlag, New York, 2002.

http://www.siam.org/journals/sidma/18-1/42713.html

${ }^{\dagger}$ Max-Planck Institute for Informatik, Stuhlsatzenhausweg 85, 66123 Saarbrücken, Germany (sunil@mpi-sb.mpg.de). Most of this research was done while this author was in the Indian Institute of Science, Bangalore, and was supported in part by the Infosys Fellowship.

${ }^{\ddagger}$ Department of Computer Science, Swiss Federal Institute of Technology, Haldeneggsteig 4, CH8092, Zürich, Switzerland (lshankar@inf.ethz.ch). 
integer, then $\Lambda(G) \leq \frac{2 n^{2}}{(k+1)^{2}}+\frac{(k-1) n}{k+1}$. When $k>5$ is an odd integer, they show that $\Lambda(G) \leq\left(1+\frac{4}{k+5}\right) n$. The inherent structural difference between graphs with odd and even edge connectivity was pointed out by Kanevsky [21] also.

In this paper, we provide upper bounds for $\Lambda(G)$ in terms of many other important parameters of graphs. We assume weighted graphs, unless otherwise specified. Multigraphs, as far as the results here are concerned, can be considered as a special case of weighted graphs, since the multiedges can be replaced by a single edge of appropriate weight without affecting the value of $\Lambda(G)$. Our only assumption about the weights is that they are positive. Note that, for the purposes of this paper, this assumption is equivalent to the assumption that the weights are at least 1 , since multiplying the weights on every edge by the same constant will not change $\Lambda(G)$. While our upper bounds are valid for weighted undirected graphs and multigraphs, in most cases, the properties in terms of which the upper bounds are stated depend only on the structure of those graphs. In other words, the radius or minimum degree in terms of which we describe the upper bounds are those of the underlying unweighted simple graph and do not depend on the weights of the edges.

There is an abundance of literature regarding the determination of $\lambda(G)$ and finding a minimum cut in $G$. The problem of enumerating all the minimum cuts is considered by many authors $[12,28,14,15]$, and various data structures are invented to efficiently represent all the minimum cuts in a graph. (The currently fastest deterministic algorithm for computing all minimum cuts in a nonnegative, real weighted graph is due to Nagamochi, Nakamura, and Ishii [26].) The fact that the performance of some of these algorithms depends on the number of minimum cuts in the graph also makes it interesting to look for tighter upper bounds for $\Lambda(G)$ when $G$ satisfies certain properties. (For example, a randomized algorithm due to Karger builds a data structure that represents all minimum cuts in $O(\Lambda(G)+n \log n)$ space.) See [14] for a brief survey of results regarding the enumeration of all minimum cuts.

The slightly different question of determining upper bounds for the number of approximate minimum cuts, i.e., those cuts having weight at most $f \lambda(G)$, where $f>1$ is a constant, is considered in [31, 22, 27, 20]. For example, Karger [22], uses probabilistic analysis to show that there are at most $O\left(n^{2 f}\right)$ cuts of the above kind in a graph of $n$ nodes. Nagamochi, Nishimura, and Ibaraki [27] show that the number of cuts of weight at most $\frac{4}{3} \lambda(G)$ is bounded above by $\left(\begin{array}{l}n \\ 2\end{array}\right)$. Henzinger and Williamson [20] show an upper bound of $O\left(n^{2}\right)$ for the number of cuts of weight at most $\frac{3}{2} \lambda(G)$, extending the arguments of [27].

1.1. Our results. Radius and diameter. If $G=(V, E)$ is a connected graph, the eccentricity of a node $v \in V$ is defined as $e(v)=\max \operatorname{distance}(v, u)$ over all the nodes $u \in V$. We define the radius of the graph $G$ as $r(G)=\min _{v \in V} e(v)$. A vertex $v$ is a central node if $e(v)=r(G)$. We define the diameter of $G$ as $d(G)=\max _{v \in V} e(v)$. (Note that, in this paper, by "distance" we mean only the distances in the underlying unweighted graph. Thus radius, eccentricity, and diameter have nothing to do with the weights.) We show that the number of minimum cuts $\Lambda(G) \leq(r+1) n-(2 r+1) \leq$ $(d+1) n-(2 d+1)$, where $G$ is a weighted graph (positive weights) and $r, d$ are the radius and diameter of $G$. As a special case, we observe that if there is a node which is a neighbor of every other node in the graph, i.e., if $r(G)=1$, then $\Lambda(G) \leq 2 n-3$. We illustrate the tightness of this bound by constructing a weighted clique $\mathcal{K}_{n}$ for which $\Lambda\left(\mathcal{K}_{n}\right)=2 n-3$.

Minimum and maximum degree. Let the minimum degree and maximum degree of $G$ be $\delta$ and $\Delta$, respectively. (Note that minimum and maximum degrees have nothing 
to do with the weights, i.e., $\delta=\min _{u \in V}|N(u)|$ and $\Delta=\max _{u \in V}|N(u)|, N(u)$ being the set of neighbors of the node $u)$. We show that $\Lambda(G) \leq\left(\frac{3 n}{2(\delta+1)}+1.5\right) n-\left(\frac{3 n}{\delta+1}+2\right)$ and $\Lambda(G) \leq \frac{(n-\Delta+3) n}{2}-(n-\Delta+2)$. Note that these bounds become significant when the involved parameters are reasonably large. Also it is easy to get an upper bound involving both $\delta$ and $\Delta$, by extending the techniques discussed in the paper.

Chordality. Let $C$ be a simple cycle of a weighted undirected graph $G$. Any edge in the induced subgraph on the nodes of $C, G[C]$, other than the cycle edges themselves, is called a chord of $C$. $C$ is called an induced cycle (or chordless cycle) ${ }^{1}$ if and only if $C$ does not have any chords. The length of the largest induced cycle in a graph $G$ is called chordality of $G$. A graph $G$ is called $k$-chordal if and only if the chordality of $G$ is at most $k$. We show that $\Lambda(G) \leq \frac{(k+1) n}{2}-k$, where $k$ is the chordality of the underlying unweighted (simple) graph corresponding to $G$. We also show the tightness of the bound by exhibiting a $k$-chordal graph $G$ for arbitrarily large $n$ such that $\Lambda(G)=\frac{(k+1) n}{2}-k$.

The word "chordality" originates from the well-known subclass of perfect graphs, the chordal graphs. A graph $G$ is chordal if and only if there is no induced cycle of length 4 or more in $G$. We define the chordality of a chordal graph to be 3. All graphs other than chordal graphs have chordality $\geq 4$. Some other important classes of graphs with low chordality value are the cocomparability graphs, chordal bipartite graphs, and weakly chordal graphs, all of which are known to be 4-chordal. It can be easily shown that asteroidal triple-free (AT-free) graphs have chordality at most 5 . Thus, by substituting the appropriate values for chordality in the above upper bound, we obtain a list of results for various special classes of graphs.

Note that $C_{n}$ (the cycle on $n$ nodes) is the graph with maximum chordality amongst all graphs on $n$ nodes. Also, it is a graph which contains the maximum number of minimum cuts possible, namely, $\left(\begin{array}{c}n \\ 2\end{array}\right)=\frac{(n+1) n}{2}-n$. (In fact, our bound given above shows that $C_{n}$ with each edge having weight $\frac{\lambda}{2}$ is the only graph which contains $\left(\begin{array}{l}n \\ 2\end{array}\right)$ minimum cuts, the weight of the minimum cut being $\lambda$ ). The fact that the maximum value of $\Lambda(G)$ is achieved by the graph of largest chordality motivates a study of the influence of chordality on $\Lambda(G)$.

Girth. Girth is the length of the smallest cycle in $G$. We show that if $G$ is an unweighted graph with girth $g$ and minimum degree $\delta$, then $\Lambda(G)<\left(\frac{n}{x+1}+1\right) n-$ $\left(\frac{2 n}{x+1}+1\right)$, where $x$ is an integer greater than $e^{-2}\left(2(\delta-1)^{\frac{g-2}{2}}-2\right)$. Note that this is in contrast with the bound in terms of chordality, the length of the largest induced cycle.

The Fiedler value. The Laplacian matrix of a graph $G$ is defined as $L=D-A$, where $A$ is the adjacency matrix and $D$ is the diagonal matrix whose $(i, i)$ th entry is the degree of the $i$ th vertex in $G$. The smallest eigenvalue of $L$ can be shown to be equal to 0 . The second smallest eigenvalue $\mu$ of $L$ is sometimes known as the Fiedler value of $G$. This is a well-studied graph parameter. It can be easily shown that if $G$ is a regular graph, then $\mu$ is equal to the gap between the two highest eigenvalues of the adjacency matrix $A$ of $G$. Various structural parameters of a graph (like diameter, vertex connectivity, vertex and edge expansion, and bisection width) are known to be related to $\mu$ and in general to the eigenvalues of $A$ or $L$. (See $[13,3,25,1]$.)

We observe that if $\mu$ is above the threshold value $1+\frac{\delta}{n-\delta}$, where $\delta$ is the minimum

\footnotetext{
${ }^{1}$ An induced cycle or a chordless cycle is often called a "hole" in the perfect graph literature. Recall that the strong perfect graph theorem characterizes perfect graphs in terms of odd holes and antiholes.
} 
degree, then all the minimum cuts in an unweighted graph $G$ are single vertex cuts. In general, if $\mu$ is the Fiedler value and $\lambda$ is the edge connectivity of $G$, we show that $\Lambda(G) \leq \frac{\left(\left\lfloor\frac{2 \lambda}{\mu}\right\rfloor+3\right)}{2} n-\left(\left\lfloor\frac{2 \lambda}{\mu}\right\rfloor+2\right)$ provided $\left\lfloor\frac{2 \lambda}{\mu}\right\rfloor<\frac{n}{3}$.

2. Preliminaries. Consider an undirected graph $G=(V, E)$ with a weight function $w: E \rightarrow \Re^{+}$. Let $U$ and $W$ be disjoint subsets of $V$. Let $E(U, W)=\{(u, v) \in$ $E: u \in U, v \in W\}$ be the set of edges between the vertices in $U$ and the vertices in $W$. Also, let $w(U, W)$ be the sum of the weights on the edges in $E(U, W)$. As mentioned in the introduction, $\lambda(G)$ denotes the weight of a minimum cut, and $\Lambda(G)$ denotes the number of minimum cuts in $G$. Let $X \subset V$. We will denote the induced subgraph on $X$ by $G[X]$.

LEMma 2.1. If $(S, \bar{S})$ is a minimum cut of a connected undirected graph $G$, then $G[S]$ and $G[\bar{S}]$ are connected.

Proof. Suppose that $G[S]$ is not connected. Let $G\left[S_{1}\right]$ be a connected component of $G[S]$, where $S_{1} \subset S$. Clearly $\left(S_{1}, \bar{S}_{1}\right)$ is a cut of $G$ and $w\left(S_{1}, \bar{S}_{1}\right)<w(S, \bar{S})$ since $E\left(S_{1}, \bar{S}_{1}\right) \subset E(S, \bar{S})$. But this is a contradiction since $(S, \bar{S})$ is assumed to be a minimum cut.

Definition 2.2. Let $(X, \bar{X})$ and $(Y, \bar{Y})$ be two cuts in a weighted undirected graph. $(X, \bar{X})$ and $(Y, \bar{Y})$ are said to cross each other if and only if all the four sets $X \cap Y, X \cap \bar{Y}, \bar{X} \cap Y$, and $\bar{X} \cap \bar{Y}$ are nonempty. Then $(X, \bar{X})$ and $(Y, \bar{Y})$ are called a crossing pair of cuts.

Lemma 2.3. A pair of cuts $(S, \bar{S})$ and $(P, \bar{P})$ do not cross if and only if $S$ (or $\bar{S}$ ) is a subset of $P$ or $\bar{P}$. (That is, $S \subseteq P, S \subseteq \bar{P}, \bar{S} \subseteq P$, or $\bar{S} \subseteq \bar{P}$.)

Proof. The proof follows from the definition of a crossing pair of cuts.

Lemma 2.4 (Bixby [7] and Dinic, Karzanov, and Lomosonov [12]). Let $(X, \bar{X})$ and $(Y, \bar{Y})$ be a crossing pair of minimum cuts in a weighted undirected graph $G$. Let $A=X \cap Y, B=\bar{X} \cap Y, C=X \cap \bar{Y}$, and $D=\bar{X} \cap \bar{Y}$. Then,

1. $w(A, B)=w(B, D)=w(D, C)=w(C, A)=\frac{\lambda(G)}{2}$;

2. $w(A, D)=w(B, C)=0$. That is, $E(A, D) \cup E(B, C)=\emptyset$.

LEMma 2.5. If $(P, \bar{P})$ and $(S, \bar{S})$ are a crossing pair of minimum cuts, then $E(P, \bar{P}) \cap E(S, \bar{S})=\emptyset$.

Proof. $E(P, \bar{P}) \cap E(S, \bar{S})=E(S \cap P, \bar{S} \cap \bar{P}) \cup E(\bar{S} \cap P, S \cap \bar{P})=\emptyset$ by Lemma 2.4.

Definition 2.6. A circular partition $\mathcal{C}=\left(U_{0}, U_{1}, U_{2}, \ldots, U_{k-1}\right)$ (where $k \geq 4$ ) of the vertices of a graph $G$ is a partition of the set of vertices $V$ of $G$ into disjoint nonempty subsets $U_{0}, U_{1}, \ldots, U_{k-1}$ such that the following hold:

1. $w\left(U_{i}, U_{i+1} \bmod k\right)=\frac{\lambda(G)}{2}$ for $0 \leq i \leq k-1$.

2. If $i \neq j+1 \bmod k$ or $i \neq j-1 \bmod k$, then $w\left(U_{i}, U_{j}\right)=0$; i.e., $E\left(U_{i}, U_{j}\right)=\emptyset$.

3. For $0 \leq i \leq k-1$, the cut $\left(U_{i}, \bar{U}_{i}\right)$-which is a minimum cut by conditions 1 and 2 -does not cross with any other minimum cut $(A, \bar{A})$ in $G$.

Definition 2.7. A cut $(A, \bar{A})$ is called a union cut with respect to a circular partition $\mathcal{C}=\left(U_{0}, U_{1}, \ldots, U_{k-1}\right)$ if and only if there exists some $i, 0 \leq i \leq k-1$, such that $A=\bigcup_{j=i}^{i+b-1} \bmod k{ }_{j}$, where $2 \leq b \leq k-2$. (Note that both $A$ and $\bar{A}$ contain at least two subsets in $\mathcal{C})$. The cut $(A, \bar{A})$ is called a subset cut with respect to $\mathcal{C}$ if and only if $A \subseteq U_{i}$ or $\bar{A} \subseteq U_{i}$ for some $i$.

Lemma 2.8. Let $\mathcal{C}=\left(U_{0}, U_{1}, \ldots, U_{k-1}\right)$ be a circular partition of $G$. Then any minimum cut $(S, \bar{S})$ of $G$ is either a union cut or a subset cut with respect to $\mathcal{C}$. Moreover, every union cut with respect to $\mathcal{C}$ is a minimum cut in $G$. 
Proof. By the definition of a circular partition, $(S, \bar{S})$ does not cross with any of the minimum cuts $\left(U_{i}, \bar{U}_{i}\right)$. Therefore, by Lemma $2.3 S \subseteq U_{i}, \bar{S} \subseteq U_{i}, S \subseteq \bar{U}_{i}$, or $\bar{S} \subseteq \bar{U}_{i}$. Suppose $(S, \bar{S})$ is not a subset cut. Then, we have $U_{i} \subseteq \bar{S}$ or $U_{i} \subseteq S$ for all $i$. Since by Lemma $2.1, G[S]$ and $G[\bar{S}]$ are connected, we infer that $(S, \bar{S})$ is a union cut. Also, that a union cut is a minimum cut follows easily from conditions 1 and 2 of the definition of a circular partition (Definition 2.6).

Lemma 2.9. Let $G$ be a weighted undirected graph. Then $G$ has a circular partition $\mathcal{C}=\left(U_{0}, U_{1}, \ldots, U_{k-1}\right)$, where $k \geq 4$, if and only if there exists a crossing pair of minimum cuts in $G$.

Proof. If there is a circular partition $\mathcal{C}=\left(U_{0}, U_{1}, \ldots, U_{k-1}\right)$ with $k \geq 4$, clearly the minimum cuts $\left(U_{0} \bigcup U_{1}, \overline{U_{0} \bigcup U_{1}}\right)$ and $\left(U_{1} \bigcup U_{2}, \overline{U_{1} \bigcup U_{2}}\right)$ cross with each other. On the other hand, if there is a crossing pair of minimum cuts in $G$, namely, $\left(S_{1}, \bar{S}_{1}\right)$ and $\left(S_{2}, \bar{S}_{2}\right)$, due to a theorem of Bixby [7] and Dinic, Karzanov, and Lomosonov [12], there exists a circular partition $\mathcal{C}=\left(U_{0}, U_{1}, \ldots, U_{k-1}\right)$ such that each of $S_{1} \cap S_{2}$, $\bar{S}_{1} \cap S_{2}, S_{1} \cap \bar{S}_{2}$, and $\bar{S}_{1} \cap \bar{S}_{2}$ equals $\bigcup_{i=a}^{b-1} U_{i}$ for appropriate choices for $a$ and $b$. The "if" part of the Lemma follows immediately from this.

For a circular partition $\mathcal{C}$ of $G$, let the partition number $p(\mathcal{C})$ be defined as the number of subsets in $\mathcal{C}$. We define the partition number of the graph $G$ as follows.

DEFINITION 2.10. The partition number $p(G)$ of a graph $G$ is defined as $p(G)=3$ if there is no circular partition for $G$. Otherwise, $p(G)=\max p(\mathcal{C})$, over all circular partitions $\mathcal{C}$ of $G$.

Note that if there is a crossing pair of minimum cuts in $G$, then $p(G) \geq 4$, by Lemma 2.9. Otherwise, $p(G)=3$.

DEFINITION 2.11. By contraction of a subset of vertices $X \subset V$, we mean replacing all the vertices in $X$ by a single vertex $x$ and adding the edges $(y, x)$ for each $y \in N(X)$, where $N(X)$ is the set of neighbors ${ }^{2}$ of $X$. The weight of the edge $(y, x)$ (where $y \in N(X))$ is assigned to be $w(y, x)=\sum_{z \in X} w(y, z)$, where $(y, z) \in E(G)$. We denote the graph obtained after the contraction operation by $G / X$. We will refer to the operation of undoing the effect of a contraction (i.e., restoring $G$ from $G / X$ ) by putting back $X$ in the place of $x$, as expanding the node $x$.

LEMma 2.12. If $(S, \bar{S})$ is a minimum cut in a weighted undirected graph $G$ such that no other minimum cut $(A, \bar{A})$ crosses with $(S, \bar{S})$, then $\Lambda(G)=\Lambda(G / S)+$ $\Lambda(G / \bar{S})-1$.

Proof. Note that since $(S, \bar{S})$ is a minimum cut, the value of the minimum cut in $G / S$ and $G / \bar{S}$ will be the same as that in $G$. First we claim that $\Lambda(G) \leq \Lambda(G / S)+$ $\Lambda(G / \bar{S})-1$. This can be seen by observing that corresponding to each minimum cut in $G$ there is a minimum cut in either $G / S$ or $G / \bar{S}$. This follows from the assumption that no minimum cut $(A, \bar{A})$ of $G$ crosses with $(S, \bar{S})$ and so exactly one of the four cases $A \supset S, A \supset \bar{S}, \bar{A} \supset S$, or $\bar{A} \supset \bar{S}$ is true by Lemma 2.3. Thus the minimum cut $(A, \bar{A})$ remains intact either in $G / S$ or $G / \bar{S}$. Also, $(S, \bar{S})$ appears in both $G / S$ and $G / \bar{S}$, which accounts for subtracting 1 . To see $\Lambda(G) \geq \Lambda(G / S)+\Lambda(G / \bar{S})-1$, observe that any minimum cut $(A, \bar{A})$ in $G / S$ or in $G / \bar{S}$ has a corresponding minimum cut in $G$. For example, consider a minimum cut $(A, \bar{A})$ in $G / S$. Without loss of generality, let the node $s$ in $G / S$ (which corresponds to the contraction of $S$ ) be in $A$. When we expand $s$, clearly the minimum cut $(A \bigcup S-\{s\}, \overline{A \bigcup S-\{s\}})$ of $G$ corresponds to the minimum cut $(A, \bar{A})$ of $G / S$. Moreover, it can be easily verified that the cuts of $G$ which correspond to the cuts of $G / S$ are distinct from the cuts of $G$ which correspond

\footnotetext{
${ }^{2} N(X)=\{u \in V-X:$ There exists a node $v \in X$ such that $(u, v) \in E\}$.
} 
to the cuts of $G / \bar{S}$ except for $(S, \bar{S})$, which is accounted for by subtracting 1 . Hence the result follows.

LEMмa 2.13. If there are no crossing pairs of minimum cuts in $G$, then $\Lambda(G) \leq$ $2 n-3$. Moreover, there exists a graph on $n$ nodes, $G_{n}$ (for every $n \geq 2$ ), such that $\Lambda\left(G_{n}\right)=2 n-3$.

Proof. If $n=2$, clearly $\Lambda(G)=1$, and the lemma is true. Assume that for all graphs with number of nodes $<n$ (where $n \geq 3$ ), the lemma is true. Consider a graph $G$ on $n$ nodes with no crossing pairs of minimum cuts. If all the minimum cuts of $G$ are singlenode cuts (i.e., of the form $(\{u\}, \overline{\{u\}})$ ), then clearly there are at most $n$ minimum cuts. Then, $\Lambda(G) \leq n \leq 2 n-3$. Otherwise, there is a minimum cut $(S, \bar{S})$ such that $|S| \geq 2$ and $|\bar{S}| \geq 2$. Let $G_{1}=G / S$ and $G_{2}=G / \bar{S}$. Also, let the number of nodes in $G_{1}$ and $G_{2}$ be $n_{1}$ and $n_{2}$, respectively. Since any minimum cut $(A, \bar{A})$ of $G$ does not cross with $(S, \bar{S})$, by Lemma 2.12 we have $\Lambda(G)=\Lambda\left(G_{1}\right)+\Lambda\left(G_{2}\right)-1$. Also, it can be easily verified that there will not be any crossing pair of minimum cuts in $G_{1}$ or $G_{2}$, since such a pair will give rise to a corresponding pair of crossing minimum cuts in $G$ also, which is a contradiction. Thus since $G_{1}$ and $G_{2}$ have $<n$ vertices, we have $\Lambda(G) \leq 2 n_{1}-3+2 n_{2}-3-1=2(n+2)-7=2 n-3$, since $n_{1}+n_{2}-2=n$. In Theorem 5.2 , we show a way to assign weights to the edges of a clique $K_{n}$ such that $\Lambda\left(K_{n}\right)=2 n-3$, illustrating the tightness of the bound given by this Lemma.

3. Partition number, $p(G)$.

Lemma 3.1. Let $G$ be a weighted undirected graph. If $(X, \bar{X})$ is a minimum cut of $G$ such that no other minimum cut crosses with $(X, \bar{X})$, then $p(G / X) \leq p(G)$.

Proof. Suppose $p(G / X)=p^{\prime}>p(G)=p$. Clearly $p^{\prime} \geq 4$ (since by definition of the partition number, $p(G)=p \geq 3$ ). Consider a circular partition $\mathcal{C}^{\prime}=\left(U_{0}, U_{1}, \ldots, U_{p^{\prime}-1}\right)$ of $G / X$. Without loss of generality, assume that the node $x$ obtained by contracting $X$ is present in $U_{0}$. We claim that $\mathcal{C}=\left(W_{0}, \ldots, W_{p^{\prime}-1}\right)$, where $W_{0}=\left(U_{0}-\{x\}\right) \cup X$ and $W_{i}=U_{i}$ for $0<i \leq p^{\prime}-1$, is a circular partition for $G$. (This will clearly contradict the assumption that $p(G / X)=p^{\prime}>p(G)$, proving the lemma).

Suppose $\mathcal{C}=\left(W_{0}, W_{1}, \ldots, W_{p^{\prime}-1}\right)$ is not a circular partition for $G$. Then, by definition of circular partition, there exists a minimum cut $(A, \bar{A})$ of $G$ which crosses with $\left(W_{i}, \bar{W}_{i}\right)$ for some $i$.

Case $1 .(A, \bar{A})$ does not cross with $\left(W_{0}, \bar{W}_{0}\right)$ but it crosses with $\left(W_{i}, \bar{W}_{i}\right)$ for some $i>0$. Since $(A, \bar{A})$ does not cross with $\left(W_{0}, \bar{W}_{0}\right)$ by Lemma 2.3 , we have (1) $A \subseteq W_{0}$, (2) $\bar{A} \subseteq W_{0},(3) A \subseteq \bar{W}_{0}$, or (4) $\bar{A} \subseteq \bar{W}_{0}$.

Case 1.1. $A \subseteq W_{0}$ or $\bar{A} \subseteq W_{0}$. Since $W_{0} \subseteq \bar{W}_{i}$, we have $A \subseteq \bar{W}_{i}$ or $\bar{A} \subseteq \bar{W}_{i}$, respectively. So, in both cases $(A, \bar{A})$ does not cross with $\left(W_{i}, \bar{W}_{i}\right)$ by Lemma 2.3, contradicting the assumption of Case 1 .

Case 1.2. $A \subseteq \bar{W}_{0}$ or $\bar{A} \subseteq \bar{W}_{0}$, i.e., $W_{0} \subseteq \bar{A}$ or $W_{0} \subseteq A$, respectively. Since $X \subseteq W_{0}, X \subseteq \bar{A}$ or $X \subseteq A$, which means that all the nodes in $X$ are on the same side of the cut $(A, \bar{A})$. Thus, the cut $\left(A^{\prime}, \overline{A^{\prime}}\right)$ of $G / X$ corresponding to $(A, \bar{A})$ is a minimum cut of $G / X$. Since $X \subseteq W_{0} \subseteq \bar{W}_{i}, X \subseteq \bar{W}_{i} \cap A$ or $X \subseteq \bar{W}_{i} \cap \bar{A}$; i.e., all the nodes in $X$ are either in $\bar{W}_{i} \cap A$ or $\bar{W}_{i} \cap \bar{A}$. But since $\left(W_{i}, \bar{W}_{i}\right)$ crosses with $(A, \bar{A})$, the sets $W_{i} \cap A, \bar{W}_{i} \cap A, W_{i} \cap \bar{A}$, and $\bar{W}_{i} \cap \bar{A}$ are nonempty. Therefore, clearly when we contract $X$ to get $G / X$, the corresponding four sets $U_{i} \cap A^{\prime}, \bar{U}_{i} \cap A^{\prime}, U_{i} \cap \overline{A^{\prime}}$, and $\bar{U}_{i} \cap \overline{A^{\prime}}$ are also nonempty. This means that in $G / X,\left(A^{\prime}, \overline{A^{\prime}}\right)$ crosses with $\left(U_{i}, \bar{U}_{i}\right)$, contradicting the assumption that $\mathcal{C}^{\prime}=\left(U_{0}, U_{1}, \ldots, U_{p^{\prime}-1}\right)$ is a circular partition for $G / X$.

Case $2 .(A, \bar{A})$ crosses with $\left(W_{0}, \bar{W}_{0}\right)$. Remember that by assumption $(A, \bar{A})$ does not 
cross with $(X, \bar{X})$. We have the following four possibilities by Lemma 2.3: (1) $A \subseteq X$, (2) $\bar{A} \subseteq X,(3) A \subseteq \bar{X}$, or (4) $\bar{A} \subseteq \bar{X}$.

Case 2.1. $A \subseteq X$ or $\bar{A} \subseteq X$. Since $X \subseteq W_{0}$, we have $A \subseteq W_{0}$ or $\bar{A} \subseteq W_{0}$, respectively, which means that by Lemma $2.3,(A, \bar{A})$ does not cross with $\left(W_{0}, \bar{W}_{0}\right)$ in both cases, contradicting the assumption of Case 2.

Case 2.2. $A \subseteq \bar{X}$ or $\bar{A} \subseteq \bar{X}$; i.e., $X \subseteq \bar{A}$ or $X \subseteq A$, which means that all the nodes in $X$ are on the same side of $(A, \bar{A})$. Thus, the cut $\left(A^{\prime}, \overline{A^{\prime}}\right)$ of $G / X$ corresponding to $(A, \bar{A})$ is a minimum cut of $G / X$. Since $X \subseteq W_{0}$, we have $X \subseteq W_{0} \cap A$ or $X \subseteq W_{0} \cap \bar{A}$, i.e., all the nodes in $X$ are completely in $W_{0} \cap A$ or $W_{0} \cap \bar{A}$. But since $\left(W_{0}, \bar{W}_{0}\right)$ crosses with $(A, \bar{A})$, the sets $W_{0} \cap A$, $\bar{W}_{0} \cap A, W_{0} \cap \bar{A}$, and $\bar{W}_{0} \cap \bar{A}$ are nonempty. Therefore, clearly when we contract $X$ to get $G / X$, the corresponding four sets $U_{0} \cap A^{\prime}, \bar{U}_{0} \cap A^{\prime}, U_{0} \cap \overline{A^{\prime}}$, and $\bar{U}_{0} \cap \overline{A^{\prime}}$ also are nonempty. This means that in $G / X,\left(A^{\prime}, \overline{A^{\prime}}\right)$ crosses with $\left(U_{0}, \bar{U}_{0}\right)$, contradicting the assumption that $\mathcal{C}^{\prime}=\left(U_{0}, U_{1}, \ldots, U_{p^{\prime}-1}\right)$ is a circular partition for $G / X$.

Thus, we infer that no minimum cut $(A, \bar{A})$ can cross with any cut $\left(W_{i}, \bar{W}_{i}\right)$ in the circular partition $\mathcal{C}=\left(W_{0}, W_{1}, \ldots, W_{p^{\prime}-1}\right)$ for $G$. But $p(\mathcal{C})=p^{\prime}>p=p(G)$, a contradiction. Thus we have $p(G / X) \leq p(G)$.

In the following lemma, we provide an upper bound for $\Lambda(G)$ in terms of the partition number. The tightness of the lemma will be established in Theorem 9.1.

Lemma 3.2. Let $G=(V, E)$ be a weighted undirected graph, where $|V|=n \geq 2$, and let the partition number $p(G) \leq p$. Then, $\Lambda(G) \leq \frac{(p+1) n}{2}-p$.

Proof. The proof is by induction on $n$. If $n=2$, by definition $p(G)=3$, and it is easy to verify that $\Lambda(G) \leq \frac{(p+1) n}{2}-p$. Now, assume that for all graphs with number of nodes $<n$ (where $n \geq 3$ ), the lemma is true. Let $G$ be a graph on $n$ nodes $(n \geq 3)$ with $p(G)=p$. If $p=3$, then by Lemma 2.9 there are no crossing pairs of minimum cuts in $G$, and hence by Lemma $2.13 \Lambda(G) \leq 2 n-3=\frac{(p+1) n}{2}-p$. Now, let $p \geq 4$. By Lemma 2.9 there exists a circular partition $\mathcal{C}=\left(U_{0}, U_{1}, \ldots, U_{p-1}\right)$ of $G$. If $p=n$, then $\left|U_{i}\right|=1$ for each $i$ and clearly $G=C_{n}$, a cycle of $n$ nodes with each edge having weight $\frac{\lambda(G)}{2}$. Therefore, it has $\left(\begin{array}{l}n \\ 2\end{array}\right)=\frac{n(n-1)}{2}=\frac{(n+1) n}{2}-n$ minimum cuts. If $p<n$, then there exists a $U_{i} \in \mathcal{C}$ such that $\left|U_{i}\right|^{2} \geq 2$. Let $G_{1}=\left(V_{1}, E_{1}\right)=G / U_{i}$ and $G_{2}=\left(V_{2}, E_{2}\right)=G / \bar{U}_{i}$ be the graphs obtained by contracting $U_{i}$ and $\bar{U}_{i}$, respectively. Since $\left|U_{i}\right| \geq 2$ and $\left|\bar{U}_{i}\right| \geq 3$ (note that this follows from $p(G) \geq 4$ ), clearly $n>\left|V_{1}\right| \geq 4$ and $n>\left|V_{2}\right| \geq 3$. Let $p_{1}=p\left(G_{1}\right)$ and $p_{2}=p\left(G_{2}\right)$. Note that by the definition of a circular partition, no minimum cut $(A, \bar{A})$ of $G$ crosses with $\left(U_{i}, \bar{U}_{i}\right)$. Hence by Lemma 2.12 we have $\Lambda(G)=\Lambda\left(G_{1}\right)+\Lambda\left(G_{2}\right)-1$. Now, by the induction assumption, $\Lambda\left(G_{j}\right) \leq \frac{\left(p_{j}+1\right) n_{j}}{2}-p_{j}$ (for $\left.j=1,2\right)$ and we have

$$
\Lambda(G) \leq \frac{\left(p_{1}+1\right) n_{1}}{2}-p_{1}+\frac{\left(p_{2}+1\right) n_{2}}{2}-p_{2}-1 .
$$

By Lemma 3.1 we have $p_{1}=p\left(G / U_{i}\right) \leq p$ and $p_{2}=p\left(G / \bar{U}_{i}\right) \leq p$ since the minimum cut $\left(U_{i}, \bar{U}_{i}\right)$ does not cross with any other minimum cut in $G$. For $n_{i} \geq 2$ and $p_{i} \leq p$ $(i=1,2)$, it is easy to verify that $\frac{\left(p_{i}+1\right) n_{i}}{2}-p_{i} \leq \frac{(p+1) n_{i}}{2}-p$. Substituting in inequality (3.1) and noting that $n_{1}+n_{2}-2=n$, we get $\Lambda(G) \leq \frac{(p+1) n}{2}-p$.

In the rest of the paper, we show that various structural parameters of a graph can influence the partition number $p(G)$. Thus by means of Lemma 3.2 we relate the number of minimum cuts, $\Lambda(G)$, with many seemingly unrelated properties of the graph. 
Remark. Please note that if $n \geq 2$ and $x \geq p$, then $\frac{(x+1) n}{2}-x \geq \frac{(p+1) n}{2}-p$. In most of the theorems below, we show that $p$ is bounded above by a function $f(y)$ of $y$, where $y$ is some parameter of $G$, thereby showing that $\Lambda(G) \leq \frac{(f(y)+1) n}{2}-f(y)$.

4. Radius and diameter. If $G=(V, E)$ is a connected graph, the eccentricity of a node $v \in V$ is defined as $e(v)=\max \operatorname{distance}(v, u)$ over all the nodes $u \in V$. We define the radius of the graph $G$ as $r(G)=\min _{v \in V} e(v)$. A vertex $v$ is a central node if $e(v)=r(G)$. We define the diameter of $G$ as $d(G)=\max _{v \in V} e(v)$. (Note that by "distance" we mean only the distances in the underlying unweighted graph. Thus radius, eccentricity, and diameter have nothing to do with the weights.)

THEOREM 4.1. If $r$ is the radius of a weighted undirected graph $G$, then $\Lambda(G) \leq$ $(r+1) n-(2 r+1)$ (where $n \geq 2)$.

Proof. Suppose there are no crossing pairs of minimum cuts in $G$. It follows by Lemma 2.13 that $\Lambda(G) \leq 2 n-3$. Since the radius is at least 1 , it is easy to verify that $\Lambda(G) \leq(r+1) n-(2 r+1)$ in this case. Otherwise, by Lemma 2.9 there exists a circular partition $\mathcal{C}=\left(U_{0}, U_{1}, \ldots, U_{p-1}\right)$ for $G$, where $p=p(G) \geq 4$. Let $x \in U_{i}$

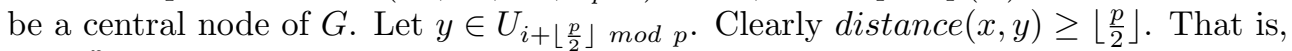
$r \geq\left\lfloor\frac{p}{2}\right\rfloor$ or $p \leq 2 r+1$. Now, by Lemma 3.2 we get $\Lambda(G) \leq(r+1) n-(2 r+1)$.

We note that the bound given by the above theorem can be tight. For example, consider $C_{2 n+1}$, the cycle on $2 n+1$ nodes. Clearly the radius of $C_{2 n+1}$ is $n$, and the number of minimum cuts $=\left(\begin{array}{c}2 n+1 \\ 2\end{array}\right)=(n+1)(2 n+1)-(2 n+1)$.

Observe that similar arguments as given for the case of the radius also hold well for the diameter. Thus,

$$
\Lambda(G) \leq(d+1) n-(2 d+1) .
$$

This can also be verified from $\Lambda(G) \leq(r+1) n-(2 r+1) \leq(d+1) n-(2 d+1)$ by noting that $d \geq r$ and $n \geq 2$.

5. Universal node. An interesting special case of Theorem 4.1 occurs when $\operatorname{radius}(G)=1$. Then, there exists a node which is adjacent to every other node of the graph. (Such a node is called a universal node.) Thus, if there is a universal node in the graph, then $\Lambda(G) \leq 2 n-3$ by Theorem 4.1. In fact, a stronger statement is true.

THEOREM 5.1. If there is a universal node $u$ in $G$, then there cannot be any crossing pairs of minimum cuts in $G$.

Proof. If there is a crossing pair of minimum cuts, then by Lemma 2.9 there is a circular partition $\mathcal{C}=\left(U_{0}, U_{1}, \ldots, U_{k-1}\right)(k \geq 4)$. Without loss of generality let $u \in U_{0}$. Clearly $u$ cannot be adjacent to any node in $U_{2}$, by the definition of circular partition, contradicting the assumption that $u$ is a universal node.

Note that in a complete graph, $K_{n}$, every node is a universal node. Thus, there are no crossing pairs of minimum cuts in a clique. Below, we show a way to assign weights to the edges of $K_{n}$ such that the number of minimum cuts $\Lambda\left(K_{n}\right)=2 n-3$, thus illustrating that the bound of Lemma 2.13 is tight. Moreover, since the radius of a clique is 1 , this is a tight example for Theorem 4.1 too. Since a complete graph is a chordal graph, the example below also illustrates the tightness of Theorem 7.2.

THEOREM 5.2. For any $n \geq 2$ and $\lambda>0$, there exists a weighted complete graph $\mathcal{K}_{n}$ such that $\lambda\left(\mathcal{K}_{n}\right)=\lambda$ and $\Lambda\left(\mathcal{K}_{n}\right)=2 n-3$. Moreover, every node $x$ of $\mathcal{K}_{n}$ defines a minimum cut $(\{x\}, \overline{\{x\}})$ of $\mathcal{K}_{n}$.

Proof. For $n=2$, it is trivial. For $n=3$, let $\mathcal{K}_{3}$ be the triangle with each edge having weight $\frac{\lambda}{2}$. Clearly $\Lambda\left(\mathcal{K}_{3}\right)=2 \cdot 3-3=3$. Also, note that every node $x$ 
in $\mathcal{K}_{3}$ defines a minimum cut $(\{x\}, \overline{\{x\}})$. Now inductively assume that there exists a weighted complete graph on $n-1$ nodes $\mathcal{K}_{n-1}(n \geq 4)$ such that $\lambda\left(\mathcal{K}_{n-1}\right)=\lambda$, $\Lambda\left(\mathcal{K}_{n-1}\right)=2(n-1)-3=2 n-5$, and every node $x$ of $\mathcal{K}_{n-1}$ defines a minimum cut $(\{x\}, \overline{\{x\}})$ in $\mathcal{K}_{n-1}$. We show how to construct a weighted complete graph $\mathcal{K}_{n}$ from $\mathcal{K}_{n-1}$ such that $\lambda\left(\mathcal{K}_{n}\right)=\lambda, \Lambda\left(\mathcal{K}_{n}\right)=2 n-3$, and every node $x$ of $\mathcal{K}_{n}$ defines a minimum cut $(\{x\}, \overline{\{x\}})$ in $\mathcal{K}_{n}$.

Let $u$ be any node of $\mathcal{K}_{n-1}$. We remove $u$ from $\mathcal{K}_{n-1}$ (along with the edges incident on it) and then add two other nodes $u^{\prime}, u^{\prime \prime}$ in its place. From each node $y$ in $\mathcal{K}_{n-1}(y \neq u)$, we add the edges $\left(y, u^{\prime}\right)$ as well as $\left(y, u^{\prime \prime}\right)$ to the new nodes and assign weights $w\left(y, u^{\prime}\right)=w\left(y, u^{\prime \prime}\right)=\frac{w(y, u)}{2}$. We also add the edge $\left(u^{\prime}, u^{\prime \prime}\right)$ with $w\left(u^{\prime}, u^{\prime \prime}\right)=\frac{\lambda}{2}$.

It is easy to see that the new graph $\mathcal{K}_{n}$ is a complete graph. Let $S=\left\{u^{\prime}, u^{\prime \prime}\right\}$. Since $(\{u\}, \overline{\{u\}})$ is a minimum cut of $\mathcal{K}_{n-1}, w(S, \bar{S})=\lambda\left(\mathcal{K}_{n-1}\right)=\lambda$. We claim that $\lambda\left(\mathcal{K}_{n}\right)=\lambda$. If not, there exists a cut $(A, \bar{A})$ in $\mathcal{K}_{n}$ such that $w(A, \bar{A})<\lambda$. If both the nodes of $S=\left\{u^{\prime}, u^{\prime \prime}\right\}$ are present on the same side of the cut $(A, \bar{A})$, then the corresponding cut in $\mathcal{K}_{n-1}$ obtained by contracting $S$ will also have weight $<\lambda$, contradicting the assumption that $\lambda\left(\mathcal{K}_{n-1}\right)=\lambda$. Therefore, without loss of generality, we can assume that $u^{\prime} \in A$ and $u^{\prime \prime} \in \bar{A}$. Then clearly $\left(u^{\prime}, u^{\prime \prime}\right) \in E(A, \bar{A})$. Also, for each $y$ in $\mathcal{K}_{n}\left(y \neq u^{\prime}\right.$ and $\left.y \neq u^{\prime \prime}\right)$, exactly one of the edges $\left(u^{\prime}, y\right)$ or $\left(u^{\prime \prime}, y\right)$ belongs to $E(A, \bar{A})$. Now recall that $w\left(y, u^{\prime}\right)=w\left(y, u^{\prime \prime}\right)=\frac{w(y, u)}{2}$ and $\sum_{y \neq u} w(y, u)=\lambda$ in $\mathcal{K}_{n-1}$. So we have $w(A, \bar{A}) \geq w\left(u^{\prime}, u^{\prime \prime}\right)+\frac{1}{2} \sum_{y \neq u^{\prime}, u^{\prime \prime}}\left(w\left(y, u^{\prime}\right)+w\left(y, u^{\prime \prime}\right)\right)=\frac{\lambda}{2}+\frac{\lambda}{2}=\lambda$, which is a contradiction to the assumption that $w(A, \bar{A})<\lambda$. Hence, $\lambda\left(\mathcal{K}_{n}\right)=\lambda$ and $(S, \bar{S})$ is a minimum cut of $\mathcal{K}_{n}$. Also, since $\mathcal{K}_{n}$ is a clique, by Theorem 5.1 no minimum cut $(A, \bar{A})$ of $\mathcal{K}_{n}$ crosses with $(S, \bar{S})$. By Lemma 2.12 we have $\Lambda\left(\mathcal{K}_{n}\right)=$ $\Lambda\left(\mathcal{K}_{n} / S\right)+\Lambda\left(\mathcal{K}_{n} / \bar{S}\right)-1$. But $\mathcal{K}_{n} / S=\mathcal{K}_{n-1}$ and $\mathcal{K}_{n} / \bar{S}=\mathcal{K}_{3}$. Thus we have $\Lambda\left(\mathcal{K}_{n}\right)=2 n-5+3-1=2 n-3$. Also it is easy to verify that every node $x$ of $\mathcal{K}_{n}$ defines a minimum cut $(\{x\}, \overline{\{x\}})$ of $\mathcal{K}_{n}$.

6. Maximum and minimum degree. The maximum degree $\Delta(G)$ (when it is reasonably high) can also constrain the number of minimum cuts $\Lambda(G)$.

THEOREM 6.1. If $\Delta$ is the maximum degree of a weighted undirected graph $G$, then $\Lambda(G) \leq \frac{(n-\Delta+3) n}{2}-(n-\Delta+2)$, where $n \geq 2$.

Proof. Suppose there are no crossing pairs of minimum cuts in $G$; then by Lemma $2.13, \Lambda(G) \leq 2 n-3 \leq \frac{(n-\Delta+3) n}{2}-(n-\Delta+2)$, which will be true if $0 \leq$ $n^{2}-(\Delta+3) n+(2 \Delta+2)$ or $0 \leq(n-\Delta-1)(n-2)$, which is true since $n \geq 2$ and $\Delta \leq n-1$. Now, if there is a crossing pair of minimum cuts in $G$, then by Lemma 2.9 there is a circular partition $\mathcal{C}=\left(U_{0}, U_{1}, \ldots, U_{p-1}\right)(p=p(G) \geq 4)$. Without loss of generality, let the maximum degree node $u \in U_{1}$. Then, $\left|U_{0} \cup U_{1} \cup U_{2}\right| \geq \Delta+1$ since every neighbor of $u$ must be in $U_{0}, U_{1}$, or $U_{2}$. Thus, $p \leq 3+(n-\Delta-1)=n-\Delta+2$ since each $U_{i}$ of the circular partition must contain at least one node. By Lemma 3.2, $\Lambda(G) \leq \frac{(n-\Delta+3) n}{2}-(n-\Delta+2)$.

Interestingly, the minimum degree of the graph can also control the number of minimum cuts.

THEOREM 6.2. If $\delta$ is the minimum degree of a weighted undirected graph $G$, then $\Lambda(G) \leq\left(\frac{3 n}{2(\delta+1)}+1.5\right) n-\left(\frac{3 n}{\delta+1}+2\right)$, where $n \geq 2$.

Proof. If there are no crossing pairs of minimum cuts in $G$, it can be easily verified that $\Lambda(G) \leq 2 n-3 \leq\left(\frac{3 n}{2(\delta+1)}+1.5\right) n-\left(\frac{3 n}{\delta+1}+2\right)$ for $n \geq 2$. Otherwise consider a circular partition $\mathcal{C}=\left(U_{0}, U_{1}, \ldots, U_{p-1}\right)(p=p(G) \geq 4)$. Group the subsets in $\mathcal{C}$ into $\left\lfloor\frac{p}{3}\right\rfloor$ triplets $\left(U_{3 i}, U_{3 i+1}, U_{3 i+2}\right)$ for $0 \leq i \leq\left\lfloor\frac{p}{3}\right\rfloor-1$. $\left|U_{3 i}\right|+\left|U_{3 i+1}\right|+\left|U_{3 i+2}\right| \geq \delta+1$ since 
each neighbor of a node $u \in U_{3 i+1}$ must be in one of the three sets in the corresponding triplet. Thus, $\left\lfloor\frac{p}{3}\right\rfloor(\delta+1) \leq n$, and the result follows by Lemma 3.2.

7. Chordality. Recall that the chordality of a graph is the length of the longest induced cycle in the graph. We provide an upper bound for $\Lambda(G)$ in terms of chordality in the following theorem. Its tightness is established in Theorem 9.1.

THEOREM 7.1. If $G$ is a weighted undirected graph with chordality $k$, then $\Lambda(G) \leq$ $\frac{(k+1) n}{2}-k$, where $n \geq 2$.

Proof. If there are no crossing pairs of minimum cuts in $G$, then by Lemma 2.13, $\Lambda(G) \leq 2 n-3 \leq \frac{(k+1) n}{2}-k$, since $k$ is at least 3 by definition and $n \geq 2$. Otherwise, consider a circular partition $\mathcal{C}$ for $G$ such that $p(\mathcal{C})=p(G)$. If $p(\mathcal{C})>k$, clearly there is an induced cycle in $G$ with length $>k$, contradicting the $k$-chordality of $G$. It follows that $p(G) \leq k$. Therefore, by Lemma $3.2, \Lambda(G) \leq \frac{(k+1) n}{2}-k$.

THEOREM 7.2. If $G$ is a weighted chordal graph, then $\Lambda(G) \leq 2 n-3$, where $n \geq 2$. Moreover, there are no crossing pairs of minimum cuts in $G$. Also, there exists a weighted chordal graph $G$, for every $n \geq 2$, such that $\Lambda(G)=2 n-3$.

Proof. Since for chordal graphs $k=3$ (by definition), $\Lambda(G) \leq 2 n-3$ follows from Theorem 7.1. If there is a crossing pair of minimum cuts in $G$, then there is a circular partition $\mathcal{C}$ for $G$ with $p(\mathcal{C}) \geq 4$ by Lemma 2.9. This immediately implies an induced cycle of length $\geq 4$, contradicting the fact that $G$ is chordal. Finally, since complete graphs are chordal graphs, the construction of Theorem 5.2 establishes the tightness of this bound.

There are some interesting special classes of graphs which can be shown to have low chordality value. We list below a few results which immeditately follow from Theorem 7.1.

Cocomparability graphs consist of graphs whose complements are comparability graphs. See [17] for the definition of a comparability graph. It can be shown that the chordality of cocomparability graphs is at most four; see, for example, [16]. Thus by Theorem 7.1 we have the following theorem.

THEOREM 7.3. If $G$ is a cocomparability graph on $n$ vertices with positive edge weights, $\Lambda(G) \leq 2.5 n-4$.

The class of weakly chordal graphs was introduced by Hayward in [19]. $G$ is defined as a weakly chordal graph if and only if neither $G$ nor the complement of $G$ contains a chordless cycle of length at least 5 . It follows from this definition that the chordality of weakly chordal graphs is at most 4 . The class of weakly chordal graphs is quite a large one, as it contains the classes of cochordal graphs, chordal bipartite graphs, permutation graphs, trapezoid graphs, tolerance graphs, 2-threshold graphs, and others. Applying Theorem 7.1, we have the following result.

THEOREM 7.4. If $G$ is a weakly chordal graph on $n$ vertices with positive weights on its edges, then $\Lambda(G) \leq 2.5 n-4$.

An independent set of three vertices such that each pair is joined by a path that avoids the neighborhood of the third is called an asteroidal triple. A graph is AT-free if it contains no asteroidal triples. AT-free graphs provide a common generalization of interval, permutation, trapezoid, and cocomparability graphs.

THEOREM 7.5. If $G$ is an AT-free graph on $n$ nodes with positive weights on the edges, then $\Lambda(G) \leq 3 n-5$.

Proof. In view of Theorem 7.1, we just have to show that an AT-free graph doesn't contain a chordless cycle of length at least 6 . Suppose it contains a chordless cycle of length 6 or more. Then clearly we can pick three points from this cycle such that they form an independent set and any two of them has a path between them, which avoids 
the neighborhood of the third. But this is not possible since the graph is assumed to be AT-free.

In fact there are many more special classes of graphs with low chordality value. The interested reader is referred to [10].

8. The stability number. The stability number $\alpha$ is defined as the size of the maximum independent set in the graph.

THEOREM 8.1.

$$
\Lambda(G) \leq(\alpha+1) n-(2 \alpha+1) .
$$

Proof. Since $\alpha$ is at least 1, if there is no crossing pair of minimum cuts in $G$, the theorem is clearly true. Otherwise it is easy to see that the partition number $p \leq 2 \alpha+1$.

9. A tight construction. We establish the tightness of Theorem 7.1 and Lemma 3.2 by the following construction.

THEOREM 9.1. For each $k \geq 3$ and $\lambda>0$, there exists an infinite family $\mathcal{G}$ of weighted undirected $k$-chordal graphs such that for each graph $G_{n} \in \mathcal{G}$ with $n$ nodes ( $n$ being an integer of the form $k+q(k-2)$ for $q=0,1, \ldots), \Lambda\left(G_{n}\right)=\frac{(k+1) n}{2}-k$, weight of the minimum cut $=\lambda$, and $p\left(G_{n}\right)=k$. Moreover, every node $u$ of $G_{n}$ defines a minimum cut $(\{u\}, \overline{\{u\}})$.

Proof. When $k=3$, the family of cliques constructed in Theorem 5.2 has the desired properties; i.e., $\lambda\left(\mathcal{K}_{n}\right)=\lambda, \Lambda\left(\mathcal{K}_{n}\right)=2 n-3, p\left(\mathcal{K}_{n}\right)=3$, and every node $x$ of $\mathcal{K}_{n}$ defines a minimum cut $(\{x\}, \overline{\{x\}})$. In the rest of the proof we assume that $k \geq 4$. First note that $G_{k}=C_{k}$ and that the cycle on $k$ nodes with each edge of weight $\frac{\lambda}{2}$ is a $k$-chordal graph with the desired properties, i.e., $\lambda\left(C_{k}\right)=\lambda, \Lambda\left(C_{k}\right)=\left(\begin{array}{l}k \\ 2\end{array}\right)=\frac{(k+1) k}{2}-k$, and $p\left(C_{k}\right)=k$. Also every node $x \in C_{k}$ defines a minimum cut.

Now we show how to inductively construct the desired family. Let $G_{n}=(V, E)$ be a $k$-chordal graph on $n$ nodes such that $\Lambda\left(G_{n}\right)=\frac{(k+1) n}{2}-k, p\left(G_{n}\right)=k$, and $\lambda\left(G_{n}\right)=$ $\lambda$. Also assume that each node $x$ in $G_{n}$ defines a minimum cut. We describe how to construct a $k$-chordal graph $G_{n^{\prime}}=\left(V^{\prime}, E^{\prime}\right)$ from $G_{n}$, where $\left|V^{\prime}\right|=n^{\prime}=n+k-2$, such that $\Lambda\left(G_{n^{\prime}}\right)=\frac{(k+1) n^{\prime}}{2}-k, \lambda\left(G_{n^{\prime}}\right)=\lambda, p\left(G_{n^{\prime}}\right)=k$, and every node of $G_{n^{\prime}}$ defines a minimum cut, thereby proving the existence of the desired family.

Construction of $G_{n^{\prime}}$ from $G_{n}$. Let $u$ be any node in $G_{n}$. Then, let $V^{\prime}=(V-$ $\{u\}) \cup P$, where $P=\left\{y_{1}, y_{2}, \ldots, y_{k-1}\right\}$ are not already present in $V$. Let $N(u)=$ $\left\{z_{1}, z_{2}, \ldots, z_{l}\right\}$ be the neighbors of $u$ in $G_{n}$. Let $E^{\prime}=\left(E-\left\{\left(u, z_{i}\right): 1 \leq i \leq\right.\right.$ $l\}) \cup\left\{\left(y_{1}, z_{i}\right): 1 \leq i \leq l\right\} \cup\left\{\left(y_{k-1}, z_{i}\right): 1 \leq i \leq l\right\} \cup\left\{\left(y_{j}, y_{j+1}\right): 1 \leq j \leq k-2\right\}$, where the weights $w\left(z_{i}, y_{1}\right)=w\left(z_{i}, y_{k-1}\right)=\frac{w\left(z_{i}, u\right)}{2}$ for $1 \leq i \leq l$ and $w\left(y_{j}, y_{j+1}\right)=\frac{\lambda}{2}$ for $1 \leq j \leq k-2$. Thus, to get $G_{n^{\prime}}$, we remove $u$ from $G_{n}$ along with the edges incident on it and add a path $\left(y_{1}, y_{2}, \ldots, y_{k-1}\right)$ with each edge of weight $\frac{\lambda}{2}$. Also each neighbor $z_{i}$ of $u$ in $G_{n}$ is now connected to $y_{1}$ and $y_{k-1}$. Moreover, the weight of $\left(z_{i}, y_{1}\right)$ and $\left(z_{i}, y_{k-1}\right)$ will be assigned half the weight of the edge $\left(z_{i}, u\right)$ in $G_{n}$. It may be noted that the contracted graphs $G_{n^{\prime}} / P=G_{n}$ and $G_{n^{\prime}} / \bar{P}=C_{k}$ with each edge having weight $\frac{\lambda}{2}$.

Claim 9.2. Let $(S, \bar{S})$ be a minimum cut of $G_{n^{\prime}}$ which crosses with the cut $(P, \bar{P})$, where $P=\left\{y_{1}, y_{2}, \ldots, y_{k-1}\right\}$. Then, exactly one of the two edges $\left(z_{i}, y_{1}\right)$ or $\left(z_{i}, y_{k-1}\right)$ $(1 \leq i \leq l)$ will belong to $E(S, \bar{S})$. (Recall that $\left\{z_{1}, z_{2}, \ldots, z_{l}\right\}$ are the nodes in $G_{n^{\prime}}$ which correspond to the neighbors of $u$ in $G_{n}$.)

Proof. First, we claim that both the nodes $y_{1}$ and $y_{k-1}$ cannot be on the same side of the minimum cut $(S, \bar{S})$. Suppose for example, $\left\{y_{1}, y_{k-1}\right\} \subseteq S$. Because all 
the edges from $P$ to $\bar{P}$ are incident on either $y_{1}$ or $y_{k-1}, E(\bar{S} \cap P, \bar{S} \cap \bar{P})=\emptyset$. (Note that $\bar{S} \cap P$ and $\bar{S} \cap \bar{P}$ will be nonempty since $(S, \bar{S})$ is assumed to cross with $(P, \bar{P})$.) Therefore the induced subgraph on $\bar{S}$ will be disconnected, which is a contradiction of Lemma 2.1 since $(S, \bar{S})$ is assumed to be a minimum cut. Now without loss of generality assume that $y_{1} \in S$ and $y_{k-1} \in \bar{S}$. Then clearly one of the two edges $\left(z_{i}, y_{1}\right)$ or $\left(z_{i}, y_{k-1}\right)$ (since both these edges exist by construction) will belong to $E(S, \bar{S})$.

Claim 9.3. $\lambda\left(G_{n^{\prime}}\right)=\lambda\left(G_{n}\right)=\lambda$ and $(P, \bar{P})$ is a minimum cut of $G_{n^{\prime}}$.

Proof. First note that the cut $(P, \bar{P})$ in $G_{n^{\prime}}$ has weight $w(P, \bar{P})=\lambda$. This is easily seen from the fact that if we contract $P$, replacing the set $P$ with the node $u$, we will get $G_{n}$ (i.e., $\left.G_{n^{\prime}} / P=G_{n}\right)$, and the cut $(P, \bar{P})$ in $G_{n^{\prime}}$ will correspond to the single vertex minimum cut $(\{u\}, \overline{\{u\}})$ in $G_{n}$. Now we will show that every cut in $G_{n^{\prime}}$ has weight at least $\lambda$, thereby establishing that $\lambda\left(G_{n^{\prime}}\right)=\lambda$ and $(P, \bar{P})$ is a minimum cut of $G_{n^{\prime}}$. Suppose $\lambda\left(G_{n^{\prime}}\right)<\lambda$. Then let $(S, \bar{S})$ be a minimum cut of $G_{n^{\prime}}$. If $S$ (or $\bar{S}$ ) is a subset of $P$ or $\bar{P}$, then one of the contracted graphs $G_{n^{\prime}} / P=G_{n}$ or $G_{n^{\prime}} / \bar{P}=C_{k}$ will contain a corresponding cut with the same value, which clearly will be a contradiction since $\lambda\left(G_{n}\right)=\lambda$ and $\lambda\left(C_{k}\right)=\lambda$. Thus by Lemma $2.3,(S, \bar{S})$ must cross with $(P, \bar{P})$ in $G_{n^{\prime}}$, which means $S \cap P, S \cap \bar{P}, \bar{S} \cap P$, and $\bar{S} \cap \bar{P}$ are nonempty. Now, by Claim 9.2, exactly one of the two edges $\left(z_{i}, y_{1}\right)$ or $\left(z_{i}, y_{k-1}\right)(1 \leq i \leq l)$ will belong to $E(S, \bar{S})$. Recall that $w\left(z_{i}, y_{1}\right)=w\left(z_{i}, y_{k-1}\right)$ and $\sum_{i=1}^{i=l} w\left(z_{i}, y_{1}\right)+\sum_{i=1}^{i=l} w\left(z_{i}, y_{k-1}\right)=w(\{u\}, \overline{\{u\}})=\lambda\left(G_{n}\right)=\lambda$. Therefore, the total contribution to the weight of $(S, \bar{S})$ due to the edges of the form $\left(y_{j}, z_{i}\right)(j=$ $1, k-1$, and $1 \leq i \leq l)$ is $\frac{\lambda}{2}$. Also, since each edge in the path defined by the nodes in $P$ has weight $\frac{\lambda}{2}$, it is clear that $w(S \cap P, \bar{S} \cap P) \geq \frac{\lambda}{2}$. Thus, considering both contributions, we infer that $w(S, \bar{S}) \geq \lambda$, contradicting the assumption that $w(S, \bar{S})<\lambda$. So we have established that $\lambda\left(G_{n^{\prime}}\right)=\lambda$, and therefore $(P, \bar{P})$ is a minimum cut of $G_{n^{\prime}}$.

Claim 9.4. No minimum cut $(S, \bar{S})$ of $G_{n^{\prime}}$ crosses with the minimum cut $(P, \bar{P})$ and $\Lambda\left(G_{n^{\prime}}\right)=\frac{(k+1) n^{\prime}}{2}-k$.

Proof. Suppose a minimum cut $(S, \bar{S})$ crosses with the minimum cut $(P, \bar{P})$ in $G_{n^{\prime}}$. Then by Claim 9.2, exactly one of the two edges $\left(z_{i}, y_{1}\right)$ or $\left(z_{i}, y_{k-1}\right)(1 \leq i \leq l)$ will belong to $E(S, \bar{S})$. Clearly both $\left(z_{i}, y_{1}\right)$ and $\left(z_{i}, y_{k-1}\right)$ are in $E(P, \bar{P})$. Thus $E(S, \bar{S}) \cap E(P, \bar{P}) \neq \emptyset$, contradicting Lemma 2.5. Therefore we infer that no minimum cut $(S, \bar{S})$ of $G_{n^{\prime}}$ can cross with $(P, \bar{P})$. Now by applying Lemma 2.12 and noting that $n^{\prime}=n+k-2$, we have

$$
\begin{aligned}
\Lambda\left(G_{n^{\prime}}\right) & =\Lambda\left(G_{n}\right)+\Lambda\left(C_{k}\right)-1 \\
& =\frac{(k+1) n}{2}-k+\frac{k(k-1)}{2}-1 \\
& =\frac{(k+1)(n+k-2)}{2}-k \\
& =\frac{(k+1) n^{\prime}}{2}-k
\end{aligned}
$$

Claim 9.5. Each node $x \in V^{\prime}$ defines a minimum cut $(\{x\}, \overline{\{x\}})$ of $G_{n^{\prime}}$.

Proof. It is easy to check that the sum of weights on edges incident on the nodes of $\bar{P}$ has not changed from what it was in $G_{n}$. Also, it is clear that for $2 \leq$ $i \leq k-2, w\left(\left\{y_{i}\right\}, \overline{\left\{y_{i}\right\}}\right)=\lambda$. Finally $w\left(\left\{y_{1}\right\}, \overline{\left\{y_{1}\right\}}\right)=w\left(y_{1}, y_{2}\right)+\sum_{i=1}^{i=l} w\left(y_{1}, z_{i}\right)$ $=\frac{\lambda}{2}+\frac{w(\{u\}, \overline{\{u\}})}{2}=\frac{\lambda}{2}+\frac{\lambda}{2}=\lambda$. The same argument also holds for $y_{k-1}$. 
Claim 9.6. $G_{n^{\prime}}$ is k-chordal.

Proof. Suppose there is an induced cycle $C$ of length $>k$ in $G_{n^{\prime}}$. We consider two cases, and show contradictions in both cases.

Case 1. $C$ contains a node $y_{i}$ from $P$ other than $y_{1}$ or $y_{k-1}$. In this case, clearly $C$ also must contain the nodes $y_{1}$ and $y_{k-1}$. Let $z_{i}$ be the neighbor of $y_{1}$ in $C$ from $\bar{P}$. Then $z_{i}$ must also be the neighbor of $y_{k-1}$ in $C$, since otherwise the edge $\left(z_{i}, y_{k-1}\right)$ will form a chord for $C$. (Note that this edge exists by construction of $G_{n^{\prime}}$.) Thus $C$ will be $\left(z_{i}, y_{1}, y_{2}, \ldots, y_{k-1}, z_{i}\right)$, a cycle of length $k$, contradicting the assumption that $|C|>k$.

Case 2. $C$ does not contain any node $y_{i}$ from $P$ other than $y_{1}$ or $y_{k-1}$. In this case clearly $C$ is an (induced) subgraph of $G\left[\bar{P} \bigcup\left\{y_{1}, y_{k-1}\right\}\right]$. We claim that $C$ must contain both the nodes $y_{1}$ and $y_{k-1}$. Otherwise, if, for example, $C$ does not contain $y_{1},|C|>k$ cannot be true, since the structure of $G\left[\bar{P} \bigcup\left\{y_{k-1}\right\}\right]$ is the same as that of $G_{n}$ (except for the weights) and $G_{n}$ is assumed to be $k$-chordal. Thus we infer that $\left\{y_{1}, y_{k-1}\right\} \subset C$. Now let $z_{i}$ and $z_{j}$ be the neighbors of $y_{1}$ in the cycle $C$. (Note that $z_{i} \neq y_{k-1}$ and $z_{j} \neq y_{k-1}$ since $y_{1}$ and $y_{k-1}$ are not adjacent in $G_{n^{\prime}}$.) Then $z_{i}$ and $z_{j}$ also must be the neighbors of $y_{k-1}$, in $C$. Otherwise, for example, if $z_{i}$ is not a neighbor of $y_{k-1}$ in $C$, clearly the edge $\left(z_{i}, y_{k-1}\right)$ (which exists by construction) will form a chord for $C$, contradicting the fact that $C$ is a chordless cycle. Now if $z_{i}$ and $z_{j}$ are the neighbors of both $y_{1}$ and $y_{k-1}$ in $C$, clearly $C=\left(y_{1}, z_{i}, y_{k-1}, z_{j}, y_{1}\right)$, a cycle of length 4 , contradicting the assumption that $|C|>k$, since $k \geq 4$ by assumption.

Claim 9.7. $p\left(G_{n^{\prime}}\right)=k$.

Proof. By Claim 9.6, the chordality of $G_{n^{\prime}}$ is $k$. Since the partition number is upper-bounded by the chordality (see the proof of Theorem 7.1), we have $p\left(G_{n^{\prime}}\right) \leq k$. Now, since by Claim 9.4, the minimum cut $(P, \bar{P})$ does not cross with any other minimum cut in $G_{n^{\prime}}$, by applying Lemma 3.1 and the induction assumption that $p\left(G_{n}\right)=k$, we get $k=p\left(G_{n}\right)=p\left(G_{n^{\prime}} / P\right) \leq p\left(G_{n^{\prime}}\right) \leq k$. Therefore, it follows that $p\left(G_{n^{\prime}}\right)=k$.

10. Girth and minimum degree. In this section we give an upper bound for $\Lambda(G)$ in terms of the girth and minimum degree in the case of unweighted graphs. The following classical results are not very difficult to prove.

LEMMA 10.1. If $(S, \bar{S})$ is a minimum cut of an unweighted undirected graph $G$, then $|S|=1$ or $|S| \geq \delta$, where $\delta$ is the minimum degree of $G$.

Lemma 10.2 (see Harary [18]). If $\delta$ is the minimum degree and $\lambda(G)=\lambda$ is the size of a minimum cut (i.e., edge connectivity) in an unweighted undirected graph $G$, then $\delta \geq \lambda$.

LEMma 10.3. Let $(X, \bar{X})$ be a minimum cut of an unweighted undirected graph $G$ with girth $g$ and minimum degree $\delta \geq 3$. Then $|X|=1$ or $|X| \geq g$.

Proof. Suppose $|X|>1$. If the induced subgraph $G[X]$ on $X$ is acyclic, then its average degree $d_{x}<2$. Then clearly $|E(X, \bar{X})| \geq\left(\delta-d_{x}\right)|X|>|X|$, since $\delta \geq 3$. By Lemma $10.1,|X| \geq \delta$. Thus $|E(X, \bar{X})|>\delta$ and this is a contradiction in view of Lemma 10.2 , since $(X, \bar{X})$ is a minimum cut. Thus, $G[X]$ contains a cycle. Clearly the cycle contains at least $g$ nodes since $g$ is the girth of $G$. It follows that $|X| \geq g$.

The following is a recent result from Alon, Hoory, and Linial [2]. (The reader may recall that the average degree of a graph is defined as the sum of degrees of the vertices divided by the total number of vertices in the graph.)

LEMma 10.4 (see [2]). The number of vertices $n$ in a graph of girth $g$ and average degree at least $d \geq 2$ satisfies $n \geq N(d, g)$, where 


$$
\begin{gathered}
N(d, 2 r+1)=1+d \sum_{i=0}^{r-1}(d-1)^{i}, \\
N(d, 2 r)=2 \sum_{i=0}^{r-1}(d-1)^{i}
\end{gathered}
$$

for integer $r \geq 1$.

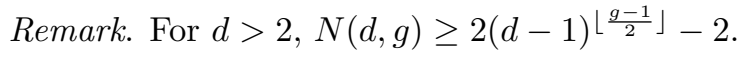

LEMma 10.5. Let $G(V, E)$ be an unweighted undirected graph with girth $g$ and minimum degree $\delta \geq 3$. If $(X, \bar{X})$ is a minimum cut of $G$, then either $|X|=1$ or $|X|>e^{-2} N(\delta, g)$, where $N(\delta, g)$ is as defined in Lemma 10.4 .

Proof. Suppose that $(X, \bar{X})$ is a minimum cut and $|X|>1$. We need to show that $|X|>e^{-2} N(\delta, g)$. Note that $e^{-2} N(\delta, 3)=e^{-2}(\delta+1)<\delta$ and $e^{-2} N(\delta, 4)=e^{-2} 2 \delta<\delta$. Therefore in view of Lemma 10.1, the present lemma is true for $g=3$ and $g=4$. Thus we can assume that $g \geq 5$.

Case 1. $g=2 r+1$. Note that since $g \geq 5, r \geq 2$. Assume for contradiction that $|X| \leq e^{-2} N(\delta, 2 r+1)$. We claim that the average degree $d_{x}$ of the induced subgraph $G[X]$ on $X$ is less than $\left(\delta-\frac{\delta-1}{r}\right)$. Suppose not. Then $d_{x} \geq\left(\delta-\frac{\delta-1}{r}\right)$. Note that since $\delta \geq 3$ and $r \geq 2, d_{x} \geq 2$. Also note that $G[X]$ is not acyclic (since $d_{x} \geq 2$ ) and its girth is at least $g=2 r+1$. Thus, Lemma 10.4 is applicable and we have

$$
\begin{aligned}
|X| & \geq N\left(\delta-\frac{\delta-1}{r}, 2 r+1\right) \\
& =1+\left(\delta-\frac{\delta-1}{r}\right) \sum_{i=0}^{r-1}\left(\delta-\frac{\delta-1}{r}-1\right)^{i} \\
& >1+\left(\delta-\frac{\delta}{r}\right) \sum_{i=0}^{r-1}\left(\delta-1-\frac{\delta-1}{r}\right)^{i} \\
& >e^{-2}+\delta\left(1-\frac{1}{r}\right) \sum_{i=0}^{r-1}(\delta-1)^{i}\left(1-\frac{1}{r}\right)^{i} \\
& >e^{-2}+\left(1-\frac{1}{r}\right)^{r} \delta \sum_{i=0}^{r-1}(\delta-1)^{i} \\
& \geq e^{-2} N(\delta, 2 r+1),
\end{aligned}
$$

which contradicts the assumption that $|X| \leq e^{-2} N(\delta, 2 r+1)$. (Note that the final step follows from the inequality $(1-x) \geq e^{-\frac{x}{1-x}}$ for $x<1$. Thus $\left(1-\frac{1}{r}\right)^{r} \geq e^{-\frac{r}{r-1}} \geq e^{-2}$ since $r \geq 2$.)

Thus we infer that $d_{x}<\delta-\frac{\delta-1}{r}$. It follows that $|E(X, \bar{X})|>\frac{\delta-1}{r}|X|$. By Lemma 10.3 we have $|X| \geq g$ and thus $|E(X, \bar{X})|>\frac{(\delta-1)(2 r+1)}{r}>\delta$, which is a contradiction in view of Lemma 10.2 since $(X, \bar{X})$ is a minimum cut. We infer that $|X|>e^{-2} N(\delta, 2 r+1)$.

Case 2. $g=2 r$. Since $g \geq 5, r \geq 3$. Assume for contradiction that $|X| \leq$ $e^{-2} N(\delta, 2 r)$. We claim that the average degree $d_{x}$ of the induced subgraph $G[X]$ on $X$ is less than $\delta-\frac{\delta-1}{r-1}$. Suppose not. Then $d_{x} \geq \delta-\frac{\delta-1}{r-1} \geq 2$ since $\delta, r \geq 3$. Also $G[X]$ is not acyclic (since $d_{x} \geq 2$ ), and its girth is at least $g=2 r$. By applying Lemma 10.4, 
we get

$$
\begin{aligned}
|X| & \geq N\left(\delta-\frac{\delta-1}{r-1}, 2 r\right) \\
& =2 \sum_{i=0}^{r-1}\left(\delta-\frac{\delta-1}{r-1}-1\right)^{i} \\
& =2 \sum_{i=0}^{r-1}(\delta-1)^{i}\left(1-\frac{1}{r-1}\right)^{i} \\
& >2\left(1-\frac{1}{r-1}\right)^{r-1} \sum_{i=0}^{r-1}(\delta-1)^{i} \\
& \geq e^{-2} N(\delta, 2 r),
\end{aligned}
$$

which is a contradiction to the assumption that $|X| \leq e^{-2} N(\delta, 2 r)$. We infer that $d_{x}<\delta-\frac{\delta-1}{r-1}$. It follows that $|E(X, \bar{X})|>\frac{\delta-1}{r-1}|X|$. Applying Lemma 10.3, we get $|E(X, \bar{X})|>\frac{\delta-1}{r-1} 2 r>\delta$, since $\delta \geq 3$. This is a contradiction in view of Lemma 10.2, since $(X, \bar{X})$ is a minimum cut. We conclude that $|X|>e^{-2} N(\delta, 2 r)$.

THEOREM 10.6. If $G$ is an unweighted undirected graph with minimum degree $\delta$ (at least 3 ) and girth $g$, then $\Lambda(G)<\left(\frac{n}{x+1}+1\right) n-\left(\frac{2 n}{x+1}+1\right)$, where $x=e^{-2} N(\delta, g)$ with $N(\delta, g) \geq 2(\delta-1)^{\left\lfloor\frac{g-2}{2}\right\rfloor}-2$, is as defined in Lemma 10.4 .

Proof. Suppose there is a circular partition $\mathcal{C}=\left(U_{0}, U_{1}, \ldots, U_{p-1}\right)$ for $G$. By Lemma $10.5,\left|U_{i}\right|=1$ or $\left|U_{i}\right|>x$. If $\left|U_{i}\right|=1$, then we claim that $\left|U_{i-1} \bmod p\right|>x$ and $\left|U_{i+1} \bmod p\right|>x$. This is because if $\left|U_{i}\right|=1$ (i.e., $\left(U_{i}, \bar{U}_{i}\right)$ defines a single-node minimum cut), then the size of the minimum cut $\lambda=\delta$. Now by the definition of circular partition, $\left|E\left(U_{i+1} \bmod p, U_{i}\right)\right|=\left|E\left(U_{i-1} \bmod p, U_{i}\right)\right|=\frac{\lambda}{2}=\frac{\delta}{2}>1$, since $\delta \geq 3$. Thus, $\left|U_{i-1 \bmod p}\right|>1$ and $\left|U_{i+1} \bmod p\right|>1$ since $G$ is an unweighted simple graph and it follows from Lemma 10.5 that $\left|U_{i-1 \bmod p}\right|>x$ and $\left|U_{i+1} \bmod p\right|>x$. Now, for each $0 \leq i \leq\left\lfloor\frac{p}{2}\right\rfloor-1,\left|U_{2 i} \bigcup U_{2 i+1}\right|>x+1$. Therefore, $\left\lfloor\frac{p}{2}\right\rfloor(x+1)<n$, and so $p<\frac{2 n}{x+1}+1$ and the theorem follows by Lemma 3.2.

We don't know whether the bound given by Theorem 10.6 is tight. But in view of the following well-known conjecture, ${ }^{3}$ it is not too bad.

ConjeCture 10.7. There exists a constant $c$ such that for all integers $g, \delta \geq 3$, there is a graph $G(g, \delta)$ of minimum degree at least $\delta$ and girth at least $g$ whose order (number of vertices) is at most $c(\delta-1)^{\left\lfloor\frac{g-1}{2}\right\rfloor}$.

Let $x=c(\delta-1)^{\left\lfloor\frac{g-1}{2}\right\rfloor}$. If Conjecture 10.7 is true, let $G_{1}, G_{2}, \ldots, G_{k}$ be $k$ copies of $G(g, \delta)$ for $k \geq g$. Now select a representative node from each of these copies. Let $x_{i}$ be the representative node from $G_{i}$. Now connect together $G_{1}, G_{2}, \ldots, G_{k}$ by a cycle of length $k$ passing through the representative nodes $x_{1}, x_{2}, \ldots, x_{k}$. Let the weight of the cycle edges $\left(x_{i}, x_{i+1}\right)$ for $i=1, \ldots, k-1$ be $\frac{\lambda}{2}$, where $\lambda$ is the edge connectivity of $G_{i}$. Let this new graph be called $G^{\prime}$. Clearly the number of minimum cuts in the new graph is at least $\left(\begin{array}{l}k \\ 2\end{array}\right)$. Thus if $n=k x$ is the total number of nodes, $\lambda\left(G^{\prime}\right)=\Omega\left(\frac{n^{2}}{x^{2}}\right)$ while the upper bound given by Theorem 10.6 is $O\left(\frac{n^{2}}{x}\right)$.

\footnotetext{
${ }^{3}$ See, for example, [9, p. 164]. Also, see [11] for a brief history of the work toward constructing such families of graphs - the so-called high girth graphs - as proposed by the conjecture. The current best result is $O\left((\delta-1)^{\frac{3 g}{4}}\right)$, achieved, for example, by the Ramanujan graphs of [24].
} 


\section{Spectral bounds for $\Lambda(G)$.}

11.1. A bound in terms of $\frac{\boldsymbol{\lambda}}{\boldsymbol{\mu}}$. Let $X$ and $Y$ be two disjoint subsets of $V$. Let $x=\frac{|X|}{n}$ and $y=\frac{|Y|}{n}$, and let $\rho$ be the distance in $G$ between $X$ and $Y$; i.e., $\rho=$ $\min _{u \in X, v \in Y}$ distance $(u, v)$. Also let $E_{X}$ denote the set of edges with both end points in the subset $X$. Let $\mu$ denote the Fiedler value, i.e., the second smallest eigenvalue of the Laplacian matrix of the graph. (See the introduction for the definition of Fiedler value.) Alon and Millman [3] proved the following result.

LEMma 11.1. $\mu n \leq \frac{1}{\rho^{2}}\left(\frac{1}{x}+\frac{1}{y}\right)\left(|E|-\left|E_{X}\right|-\left|E_{Y}\right|\right)$.

Let $(X, \bar{X})$ be a cut of $G$. Then, the following corollary to the above lemma is useful for us.

Corollary 11.2. If $(X, \bar{X})$ is any cut in $G$, then $\mu \leq\left(\frac{1}{|X|}+\frac{1}{|\bar{X}|}\right)|E(X, \bar{X})|$, where $E(X, \bar{X})$ is the set of edges between the disjoint sets $X$ and $\bar{X}$.

LEMMA 11.3. If $\mu$ is the second smallest eigenvalue of the Laplacian matrix $L$ of an unweighted undirected graph $G$, then for any minimum cut $(S, \bar{S}),|S|>\frac{n}{2}$ or $|S| \leq\left\lfloor\frac{2 \lambda}{\mu}\right\rfloor$, where $\lambda=\lambda(G)$ is the edge connectivity of $G$.

Proof. Suppose $|S| \leq \frac{n}{2}$. By Corollary 11.2, $\mu \leq\left(\frac{1}{|S|}+\frac{1}{n-|S|}\right)|E(S, \bar{S})| \leq \frac{2 \lambda}{|S|}$ since $(S, \bar{S})$ is a minimum cut. Since $|S|$ is an integer, we have $|S| \leq\left\lfloor\frac{2 \lambda}{\mu}\right\rfloor$.

COROLlary 11.4. $\left\lfloor\frac{2 \lambda}{\mu}\right\rfloor \geq 1$.

Proof. Let $(S, \bar{S})$ be a minimum cut with $|S| \leq|\bar{S}|$. Thus $|S| \leq \frac{n}{2}$. So, by Lemma $11.3,1 \leq|S| \leq\left\lfloor\frac{2 \lambda}{\mu}\right\rfloor$.

THEOREM 11.5. Let $G$ be an unweighted undirected graph with $\lambda(G)=\lambda$ and let $\mu$ be the second smallest eigenvalue of the Laplacian matrix of $G$. If $\left\lfloor\frac{2 \lambda}{\mu}\right\rfloor<\frac{n}{3}$, then $\Lambda(G) \leq \frac{\left(\left\lfloor\frac{2 \lambda}{\mu}\right\rfloor+3\right)}{2} n-\left(\left\lfloor\frac{2 \lambda}{\mu}\right\rfloor+2\right)$.

Proof. Let $\gamma=\left\lfloor\frac{2 \lambda}{\mu}\right\rfloor$. Suppose there are no crossing pairs of minimum cuts in $G$. Then by Lemma $2.13, \Lambda(G) \leq 2 n-3 \leq \frac{(\gamma+3)}{2} n-(\gamma+2)$, since $\gamma \geq 1$ (by Corollary 11.4). Otherwise, by Lemma 2.9, there exists a circular partition $\mathcal{C}=\left(U_{0}, U_{1}, \ldots, U_{p-1}\right)$ of $G$ with $p=p(G) \geq 4$. We claim that $p \leq \gamma+2$. Suppose $p>\gamma+2$. We will show a contradiction. Let $U_{j}$ be the subset in $\mathcal{C}$ such that $\left|U_{j}\right|=\max _{i}\left|U_{i}\right|$. We will show first that $\left|U_{i}\right| \leq \gamma$ for all $i \in\{(j+1) \bmod p$, $(j+2) \bmod p, \ldots,(j+\gamma+1) \bmod p\}$. If $\left|U_{j}\right| \leq \gamma$, this is clearly true since $\left|U_{j}\right|=\max _{i}\left|U_{i}\right|$ by assumption. Remembering that $\left(U_{j}, \bar{U}_{j}\right)$ is a minimum cut, by Lemma 11.3, if $\left|U_{j}\right|>\gamma$, then $\left|U_{j}\right|>\frac{n}{2}$. Therefore, $\left|V-U_{j}\right|<\frac{n}{2}$. Thus, for $i \in\{(j+1) \bmod p,(j+2) \bmod p, \ldots,(j+\gamma+1) \bmod p\},\left|U_{i}\right| \leq\left|V-U_{j}\right|<\frac{n}{2}$ and since $\left(U_{i}, \bar{U}_{i}\right)$ is a minimum cut, by Lemma $11.3,\left|U_{i}\right| \leq \gamma$, as required.

Now let $k$ be the smallest integer such that $\left|\bigcup_{i=j+1}^{j+k} U_{i}\right|>\gamma$. (Note that $\gamma+1 \geq$ $k \geq 2$.) Let $X=\bigcup_{i=j+1}^{j+k} U_{i}$. Note that since $\left|X-U_{j+k}\right| \leq \gamma$ and $\left|U_{j+k}\right| \leq \gamma$, we have

$$
|X| \leq 2 \gamma<\frac{2 n}{3}
$$

But $(X, \bar{X})$ is a minimum cut by Lemma 2.8. Since $|X|>\gamma$, we have by Lemma 11.3 that $|X|>\frac{n}{2}$. That is, $|\bar{X}|<\frac{n}{2}$. It follows from inequality (11.1) that $\gamma<\frac{n}{3}<|\bar{X}|<$ $\frac{n}{2}$, which is again a contradiction by Lemma 11.3 since $(\bar{X}, X)$ is a minimum cut. Thus we infer that $p \leq \gamma+2$ and hence by Lemma 3.2, the theorem follows. (Please note that if $n \geq 2$ and $f \geq p$, then $\frac{(f+1) n}{2}-f \geq \frac{(p+1) n}{2}-p$.) 
The above bound is interesting for certain ranges of $\lambda$ and $\mu$, for example, when $\lambda$ is relatively small and $\mu$ is not too small, say, not $O\left(\frac{1}{n}\right)$. It may be noted that restricting $\lambda$ to be bounded above by a constant doesn't imply that the value of $\mu$ also will be small. In fact there are $\delta$-regular graphs for which the value of $\mu$ can be as high as $\Omega(\sqrt{\delta})$.

11.2. When $\boldsymbol{\mu}$ is large. We observe that if $\mu$ is above a threshold value, then all the minimum cuts are single-vertex cuts, i.e., cuts of the form $(\{x\}, \overline{\{x\}})$, where $x$ is a node. This is captured in the following theorem.

THEOREM 11.6. Let $\mu$ be the second smallest eigenvalue of the Laplacian $L$ of an unweighted undirected graph $G$. If $\mu>1+\frac{\delta}{n-\delta}$, where $\delta$ is the minimum degree, then all the minimum cuts in $G$ are single-vertex minimum cuts.

Proof. If there is a minimum cut $(S, \bar{S})$ with $|S| \leq|\bar{S}|$ and $|S| \neq 1$, then from Lemma 10.1, we have $|S| \geq \delta$. By Corollary 11.2, $\mu \leq\left(\frac{1}{|S|}+\frac{1}{|\bar{S}|}\right) \lambda$. But this is a contradiction when $\mu>1+\frac{\delta}{n-\delta}$ since $\lambda \leq \delta$ by Lemma 10.2 .

The threshold given by the above theorem is tight. For example, it can be verified that for the graph $C_{4}$, the cycle graph on four nodes, $n=4, \delta=2$, and $\mu=2$. Thus for $C_{4}, 1+\frac{\delta}{n-\delta}=\mu$, but it has minimum cuts which are not single-vertex cuts. In fact it is also possible to construct such examples with a larger number of nodes.

\section{REFERENCES}

[1] N. Alon, Eigenvalues and expanders, Combinatorica, 6 (1986), pp. 83-96.

[2] N. Alon, S. Hoory, And N. Linial, The Moore bound for irregular graphs, Graphs Combin., 18 (2002), pp. 53-57.

[3] N. Alon AND V. D. Millman, $\lambda_{1}$, isoperimetric inequalities for graphs, and superconcentrators, J. Combin. Theory Ser. B, 38 (1985), pp. 73-88.

[4] M. Ball And J. Provan, Calculating bounds on reachability and connectedness in stochastic networks, Networks, 13 (1983), pp. 253-278.

[5] J. Provan AND M. BALL, The complexity of counting cuts and of computing the probability that a graph is connected, SIAM J. Comput., 12 (1983), pp. 777-788.

[6] M. Ball and J. Provan, Computing network reliability in time polynomial in the number of cuts, Oper. Res., 32 (1984), pp. 516-521.

[7] R. BIXBY, The minimum number of edges and vertices in a graph with edge connectivity $n$ and $m$ n-bonds, Networks, 5 (1975), pp. 253-298.

[8] F. Boesch, Synthesis of reliable networks - a survey, IEEE Trans. Reliability, R-35 (1986), pp. 240-246.

[9] B. Bollobás, Extremal Graph Theory, London Math. Soc. Monogr. 11, Academic Press, London, New York, 1978.

[10] A. Brandstädt, V. B. Le, and J. P. Spinrad, Graph Classes: A Survey, SIAM, Philadelphia, 1999.

[11] L. S. Chandran, A high girth graph construction, SIAM J. Discrete Math., 16 (2003), pp. 366370.

[12] E. Dinic, A. Karzanov, and M. Lomosonov, The structure of a system of minimal edge cuts of a graph, in Studies in Discrete Optimization, Nauka, Moscow, 1976, pp. 290-306 (in Russian).

[13] M. FiedLER, The algebraic connectivity of graphs, Czechoslovak Math J., 23 (1973), pp. 298305.

[14] L. FleISCHER, Building chain and cactus representations of all minimum cuts from Hao-Orlin in the same asymptotic run time, J. Algorithms, 33 (1999), pp. 51-72.

[15] H. Gabow, A matroid approach to finding edge connectivity and packing arborescences, J. Comput. System Sci., 50 (1995), pp. 259-273.

[16] T. Gallai, Transitiv orientierbare graphen, Acta. Math. Acad. Sci. Hungar., 18 (1967), pp. $25-66$.

[17] M. C. Golumbic, Algorithmic Graph Theory and Perfect Graphs, Academic Press, New York, 1980.

[18] F. Harary, Graph Theory, Addison-Wesley, Reading, MA, 1969. 
[19] R. B. HaYward, Weakly triangulated graphs, J. Combin. Theory Ser. B, 39 (1985), pp. 200-208.

[20] M. Henzinger and D. Williamson, On the number of small cuts, Inform. Process. Lett., 59 (1996), pp. 41-44.

[21] A. Kanevsky, Graphs with Odd and Even Edge Connectivity Are Inherently Different, Tech. Rep. TAMU-89-10, Texas A \& M University, College Station, TX, 1989.

[22] D. KARGer, Random sampling in cut, flow, and network design problems, Math. Oper. Res., 24 (1999), pp. 383-413.

[23] J. Lehel, F. Maffray, and M. Preissmann, Graphs with largest number of minimum cuts, Discrete Appl. Math., 65 (1996), pp. 387-407.

[24] A. Lubotzky, R. Phillips, and P. SARnak, Ramanujan graphs, Combinatorica, 8 (1988), pp. 261-271.

[25] B. MohaR, Eigenvalues, diameter, and mean distance in graphs, Graphs. Combin., 7 (1991), pp. 53-64.

[26] H. Nagamochi, S. NakAmura, AND T. IshiI, Constructing a cactus for minimum cuts of a graph in $o\left(m n+n^{2} \log n\right)$ time and o $(m)$ space, Inst. Electron. Inform. Comm. Eng. Trans. Fundamentals, E86-D (2003), pp. 179-185.

[27] H. Nagamochi, K. Nishimura, and T. Ibaraki, Computing all small cuts in an undirected network, SIAM J. Discrete Math., 10 (1997), pp. 469-481.

[28] D. NaOr And V. V. VAZIRANi, Representing and enumerating edge connectivity cuts in RNC, in Proceedings of the Second Workshop on Algorithms and Data Structures, Lecture Notes in Comput. Sci. 519, Springer-Verlag, New York, 1991, pp. 273-285.

[29] J. Picard And M. Queyranne, On the structure of all minimum cuts in a network and applications, Math. Programming Stud., 13 (1980), pp. 8-16.

[30] J. Provan, Bounds on the reliability of networks, IEEE Trans. Reliability, R-35 (1986), pp. 260268.

[31] V. V. Vazirani and M. Yannakakis, Suboptimal cuts: Their enumeration, weight, and number, in Proceedings of the 19th International Colloquium on Automata, Languages and Programming, Lecture Notes in Comput. Sci. 623, Springer-Verlag, New York, 1992, pp. 366377. 